\title{
Fritz Böhle, Eva Senghaas-Knobloch (Hrsg.): Andere Sichtweisen auf Subjektivität. Impulse für kritische Arbeitsforschung
}

\author{
Springer VS, Heidelberg 2020
}

\author{
Wibke Derboven ${ }^{1}$ \\ Online publiziert: 13 . Mai 2020 \\ (c) Der/die Autor(en) 2020
}

Impulse für die Arbeitsforschung sind im Zuge des tiefgreifenden Wandels der Erwerbsarbeit sehr zu begrüßen und von hohem Wert. In diesem Sammelband stellt die Arbeitsforschung ihren traditionellen Analyserahmen selbstreflexiv und interdisziplinär auf den Prüfstand und entwickelt neue Sichtweisen, wobei die Subjektivität im Zentrum steht. Hier diskutieren Arbeitsforscher und Arbeitsforscherinnen mit soziologischem, psychologischem und psychoanalytischem Gewicht verschiedene und je eigene Sichtweisen auf Subjektivität. Dabei teilen sie die Überzeugung, dass Subjektivität mehr Gewicht, aber auch neue Sichtweisen braucht und verfolgen gemeinsam - und im Versuch sich wechselseitig zu verstehen - das Ziel, einen neuen Bezugsrahmen für die kritische Arbeitsforschung zu skizzieren. Alle Autorinnen und Autoren setzten sich in ihren Beiträgen kritisch mit dem Wandel der Arbeit auseinander, beschreiben die damit verbundenen, schwerpunktmäßig negativen Phänomene und schließen mit einem Plädoyer für eine bestimmte Sichtweise auf Subjektivität, die nach ihrer je eigenen Auffassung ein Bestandteil kritischer Arbeitsforschung sein sollte. Dabei streifen sie Erkenntnisse der bisherigen, sich gegen tayloristische Formen von Arbeit gerichtete Arbeitsforschung, beziehen sich auf gewichtige Theoretiker wie beispielsweise und um nur einige $\mathrm{zu}$ nennen: Theodor W. Adorno, Pierre Bourdieu, Norbert Elias, Michel Foucault, Erich Fromm, Jürgen Habermas, Karl Marx und ziehen ihre Schlussfolgerungen aus eigenen, aktuellen empirischen Studien in den Bereichen: Pflege, Automobilindustrie, Energiewirtschaft und Plattformökonomie. Unbedingt zu erwähnen ist, dass dieses Werk kein Schnellschuss ist, sondern ,das Ergebnis eines sich über mehrere

Wibke Derboven

derboven@tuhh.de

1 Technische Universität Hamburg, Hamburg, Deutschland
Jahre erstreckenden Diskussionsprozesses" (S. 4) zwischen den Autorinnen und Autoren. Die Leserinnen und Leser dürfen geballte kollektive Expertise erwarten und bekommen hervorragende Impulse, eine eigene fundierte Haltung zum Wandel der Arbeit und zu nützlichen (kritischen) Analysekriterien zu entwickeln.

Nach einer Einführung durch die Herausgebenden werden in sieben Beiträgen verschiedene Perspektiven auf aktuelle Arbeitsbedingungen eingenommen und notwendige Neuorientierungen für eine kritische Analyse derselben erörtert. Fritz Böhle kritisiert im Beitrag „Humane Arbeit als geistige Arbeit?" die unhinterfragte normative Referenz, dass humane Arbeit geistige Arbeit ist. Mit Betonung der zunehmenden Entkörperlichung von Arbeit und Technisierung von geistiger Arbeit plädiert er dafür, den Blick auf das Nicht-Messbare, Nicht-Objektivierbare neu auszurichten. Guido Becke betont im Beitrag „Relationale Subjektivität in subjektivierten Arbeitsverhältnissen“ die soziale Verfasstheit der Subjektivität und zeigt, dass Sozialität nicht als Einschränkung der Autonomie, sondern zunehmend als Bewältigungsquelle im Umgang mit den neuen subjektivierten Anforderungen an Arbeit zu begreifen ist. Birgit Volmerg blickt unter dem Titel ,Wie brauchbar sind rollentheoretische Konzeptionen in flexibilisierten Arbeitsund Lebenswelten?" kritisch auf die Nützlichkeit der bisher für eine kritische Arbeitsanalyse wichtigen rollentheoretischen Konzepte und sieht eine Alternative im Konzept der „Sorge um sich“, wie es Foucault mit Rekurs auf die antike Lebenspraxis der Polis ausgearbeitet hat. Werner Fricke kritisiert im Beitrag „Aus der Subjektivierungsfalle zum handlungsfähigen Akteur - Das Versprechen der Aktionsforschung" die derzeitige Subjektivierung von Arbeit als „Illusion der Selbstbestimmung“ (S. 74). Er plädiert dafür selbstbestimmtes Handeln von Subjekten, das für ihn nur im Rahmen von solidarisch-kollektivem Handeln von Gruppen erreichbar ist, mithilfe der Aktionsforschung wieder 
$\mathrm{zu}$ beleben und $\mathrm{zu}$ stabilisieren. Im Beitrag „Herrschaftsverhältnisse und Wissensformen - Kritische Betrachtung eines Umbruchs bei der Wissensvermittlung in Unternehmen" kritisiert David Faure die Vorherrschaft und zunehmende Hinwendung zum expliziten Wissen, verweist auf den Zusammenhang zu neuen Formen von Unternehmensherrschaft und arbeitet die hohe Bedeutung von impliziten - auch kollektiven impliziten - Wissensformen für gute Arbeitsbedingungen heraus. Christel Kumbruck und Eva Senghaas-Knobloch kritisieren im Beitrag „,Die Grenzen instrumenteller Verfügbarkeit von Subjektivität - Einsichten aus der Arbeitswelt der Pflege“ die Vernachlässigung der Subjektivität in der auf Zeitökonomie ausgelegten Arbeitsorganisation in der Pflege und arbeiten die besondere Notwendigkeit der Subjektivität in dieser interaktiven Arbeit heraus, zeigen aber auch, dass der Versuch ihrer instrumentellen Verfügbarkeit unbeabsichtigte negative Folgen nach sich zieht. Stephan Voswinkel kritisiert in seinem Artikel „Entfremdung und Aneignung in der Arbeit“, dass die aktuelle Arbeitsforschung den Begriff der Entfremdung als kritischen Analysebegriff und als passendes Deutungsmuster verwirft und plädiert für die Stärkung und Neufassung des Begriffs. Er wendet sich gegen individualistische und verkürzte Interpretationen von Entfremdung und arbeitet kollektive Aspekte, die hohe Bedeutung des Sinns und darüber hinaus auch emanzipatorische Potentiale von Entfremdung heraus. Thomas Leithäuser erörtert im Beitrag „SubjektObjekt-Relationen in der Sozialisation, in der Arbeit und im Alltag“ den Nutzen einer sozialpsychologischen Untersuchung der Subjekt-Objekt-Relationen zwischen Menschen und Dingen sowie zwischen Menschen untereinander und beschreibt die Entgrenzung von Subjekt-Objekt-Relationen durch Digitalisierung.

Was für ein gewichtiges, sehr lesenswertes, zu empfehlendes Buch: zum Eintauchen in die Welt der Arbeitsforschung, zum Reflektieren der eigenen Arbeit und als Hilfestellung, eine eigene (kritische) Haltung zur aktuellen Gestaltung von Arbeit zu entwickeln. Alle, die sich mit der Erforschung und Gestaltung von Arbeit beschäftigen, können von dem Buch profitieren und Interessantes finden: zur Geschichte und Tradition der kritischen Arbeitsforschung, zu ihrem bisherigen Gegenspieler, der tayloristischen Arbeitsorganisation, zu ihrem aktuellen Gegenspieler, zu den
Phänomenen, die der Wandel der Arbeit mit sich bringt, zu erneuerten, an den Wandel angepassten Kriterien einer kritischen Arbeitsanalyse, aber auch zu anthropologischen Konstanten. Insbesondere erwähnen möchte ich an dieser Stelle die Bedeutung für eine Überprüfung der aktuellen Interpretation des Konzepts der New Work (Frithjof Bergmann), das als Oberbegriff der Transformation der Arbeitswelt weit verbreitet ist. Insbesondere in der Startup-Szene vielfach in einer Engführung genutzt, kann das Buch dazu beitragen zwischen illusionärer und emanzipatorischer Handlungsfähigkeit zu unterscheiden. Gewünscht hätte ich mir eine zusammenfassende Beurteilung, die die Gemeinsamkeiten, aber auch das Trennende aller Beiträge zusammenfassend darstellt. Dies müssen die Leserinnen und Leser nun selbst leisten. Für mich ist besonders bemerkenswert, welch hohen Stellenwert das Kollektive in diesem Sammelband über Subjektivität einnimmt. In diesem Sammelband entfalten sich - in meiner subjektivierten Lesart - intersubjektive und interaktive Arbeitssubjekte in ihrer dynamischen Verfasstheit, ihren Bedürfnissen und ihren unverzichtbaren, nie vollständig objektivierbaren Fähigkeiten, die sich (erst) im Austausch mit anderen Menschen und der gegenständlichen Umwelt subjektivieren und (erst) im demokratischen Dialog handlungsfähig werden.

Funding Open Access funding provided by Projekt DEAL.

Open Access Dieser Artikel wird unter der Creative Commons Namensnennung 4.0 International Lizenz veröffentlicht, welche die Nutzung, Vervielfältigung, Bearbeitung, Verbreitung und Wiedergabe in jeglichem Medium und Format erlaubt, sofern Sie den/die ursprünglichen Autor(en) und die Quelle ordnungsgemäß nennen, einen Link zur Creative Commons Lizenz beifügen und angeben, ob Änderungen vorgenommen wurden.

Die in diesem Artikel enthaltenen Bilder und sonstiges Drittmaterial unterliegen ebenfalls der genannten Creative Commons Lizenz, sofern sich aus der Abbildungslegende nichts anderes ergibt. Sofern das betreffende Material nicht unter der genannten Creative Commons Lizenz steht und die betreffende Handlung nicht nach gesetzlichen Vorschriften erlaubt ist, ist für die oben aufgeführten Weiterverwendungen des Materials die Einwilligung des jeweiligen Rechteinhabers einzuholen.

Weitere Details zur Lizenz entnehmen Sie bitte der Lizenzinformation auf http://creativecommons.org/licenses/by/4.0/deed.de. 